\title{
Biologia della Cellula Staminale: potenza e differenziazione
}

\section{Lombardi}

Dottore in Biotecnologie Medico-Diagnostiche, Studente presso il Corso Magistrale in Terapie Biologiche e Cellulari in Medicina, CdL in Biotecnologie Mediche, Medicina, Università degli Studi di Firenze.

Tirocinante del Laboratorio Interdipartimentale di Nefrologia Cellulare e Molecolare diretto dalla Professoressa P. Romagnani, Facoltà di Medicina e Chirurgia, Università degli Studi di Firenze

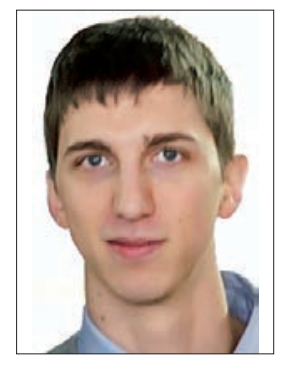

\section{Introduzione}

Una delle caratteristiche sicuramente più note e che distingue funzionalmente una cellula staminale rendendola di così ampio interesse in campo medico, specialmente a fini terapeutici, è la sua capacità di dar luogo, in seguito a specializzazione o commitment, a cellule con differenti funzioni e specializzazioni. Questa capacità è definita dal concetto di potenza.

Con questo termine è indicata la possibilità di tali cellule di andare incontro a un differenziamento multilineare che, a seconda del tipo di cellula staminale, può essere a più ampio o ristretto raggio. $\grave{E}$ ad esempio noto che lo zigote sia totipotente, ossia in grado di dar luogo sia a ogni tipo cellulare che costituirà i tre foglietti embrionali da cui originano poi i vari tessuti dell'embrione stesso (Ectoderma, Endoderma e Mesoderma), sia a quelle cellule che costituiranno gli annessi embrionali e che porteranno, ad esempio, allo sviluppo del sacco vitellino. Una cellula staminale è altresì definita pluripotente qualora possa dar luogo ai soli 3 foglietti embrionali e, conseguentemente, ai vari tessuti che costituiranno il nuovo individuo. È questa una caratteristica tipica delle cellule staminali embrionali (ESC), ossia di quelle cellule che costituiscono la Inner Cell Mass della blastocisti e che, se prelevate tra il $4^{\circ}$ e il $7^{\circ}$ giorno di gestazione e reinoculate in una diversa blastocisti, sono capaci di integrarsi in tutti i tessuti embrionali, evento che prova la loro multipotenza (1). Anche in questo caso, come per lo zigote, il processo di differenziazione non è ancora ristretto a uno specifico ambito tissutale. Una cellula staminale è invece multipotente qualora sia già committed, ossia possa dar luogo solo alle cellule che fanno parte del tessuto di appartenenza della cellula staminale stessa. Queste sono le cellule staminali fetali o adulte (ASC), le quali assumono la funzione di turnover fisiologico e mantenimento dell'omeostasi del tessuto in cui risiedono, o di riparazione in seguito ad insulto, motivo per cui sono quiescenti ma sempre pronte all'attivazione e alla sostituzione. Le cellule staminali adulte sono state individuate in tessuti derivanti da tutti e $3 \mathrm{i}$ foglietti embrionali e sono perciò definite come cellule indifferenziate residenti in un tessuto specializzato adulto (1).

Risulta quindi evidente, da tale descrizione, come quello che è lo sviluppo di un nuovo organismo sia caratterizzato da una graduale perdita di potenzialità differenziativa delle varie categorie di cellule staminali, in quanto da queste originano via via cellule sempre maggiormente specializzate. Questo processo, denominato restrizione, non è però così irreversibile come si pensava fino a pochi anni fa. Esso appare difatti abbastanza plastico, come dimostrato grazie alla riprogrammazione di cellule somatiche differenziate, le quali possono essere revertite ad un fenotipo e ad una funzionalità tipiche delle cellule staminali embrionali: si parla di iPS, o Induced Pluripotent Stem Cells (1). 


\section{Fattori chiave per la toti, pluri o multipotenza}

Nel gioco della potenzialità differenziativa intervengono molti e diversi fattori a seconda che si parli di cellule staminali embrionali o adulte, anche se sono comunque individuabili dei coefficienti comuni ad entrambi i tipi di entità staminali. Un ruolo di primo piano nel mantenere l'ampia capacità differenziativa che una cellula staminale possiede è svolto da Oct- 4 , fattore trascrizionale che porta all'espressione di numerosi geni, il cui calo di concentrazione, ad esempio, induce le cellule a differenziare in endoderma extraembrionico, mentre un suo aumento è correlato al commissionamento delle ESC in mesoderma o ectoderma: se quindi non è espresso costantemente la cellula differenzia.

Altro fattore trascrizionale di notevole interesse e rilievo è Nanog, la cui espressione è direttamente correlata al mantenimento dello stato indifferenziato o parzialmente commissionato della cellula, mentre una diminuzione della sua presenza è indissolubilmente legata al differenziamento dell'entità staminale. Come Oct-4, quindi, Nanog è usato come marcatore per l'individuazione di cellule staminali. Queste due proteine è stato dimostrato che collaborano nel mantenere un fenotipo pluripotente nelle cellule staminali embrionali, pur essendo l'una indipendente dall'altra (1).

Tali due geni sono fondamentali anche per la riprogrammazione di cellule somatiche adulte al fine di ottenere le iPS. Nel Paper (2) in questione, pubblicato nel 2006, per attuare la riprogrammazione delle cellule sono stati utilizzati 4 vettori retrovirali che hanno forzato l'espressione di 4 geni: c-Myc, Sox-2, KLF-4 ed Oct-4. La loro espressione ha poi determinato l'espressione endogena di Nanog e dello stesso Oct-4. In questo modo è stato stabilito e poi mantenuto lo stato di pluripotenza (3).

Nel mantenimento della pluripotenza risulta fondamentale anche la metilazione (aggiunta di gruppi $\mathrm{CH}_{3}$ ) del DNA. Questa modificazione covalente rende difatti meno accessibili geni lineage specifici indirizzando così verso il differenziamento della cellula. Analogamente è di primaria importanza lo stato cromatinico: il DNA può infatti essere sia in forma non compatta (eucromatina), stato funzionale sia alla duplicazione del materiale genetico che alla trascrizione, che in forma eterocromatinica, la quale essendo più compatta inibisce l'espressione genica. La cromatina in conformazione prevalentemente aperta rende accessibili vari e diversi programmi di differenziazione, mentre il commissionamento di una cellula staminale è associato invece a condensazione cromatinica e, di conseguenza, a un più ristretto profilo di espressione (1).

\section{Le Staminali Adulte: specializzazione e ruolo della nicchia staminale}

Le ASC rappresentano una piccola sottopopolazione del compartimento proliferante di un dato tessuto. Sono cellule relativamente indifferenziate che, quando si dividono, mantengono le dimensioni della popolazione staminale e, allo stesso tempo, danno luogo a una progenie che duplicandosi differenzia in primis in una popolazione di transizione, mentre in un secondo tempo assume le caratteristiche funzionali di cellule completamente differenziate.

Questa graduale specializzazione avviene sia per il ricambio omeostatico di cellule morte del tessuto, che per la sostituzione di cellule danneggiate in seguito ad insulto. Tale sostituzione segue uno schema gerarchico che prende origine da una singola cellula staminale la quale si divide asimmetricamente dando poi luogo a una popolazione di transizione che inizia ad amplificarsi e, grazie a una serie di divisioni progressive, va incontro al restringimento del lineage. Ciò permette la creazione di una sorta di "gerarchia staminale": le cellule che differenziano vanno via via perdendo le loro caratteristiche di staminalità, mentre aumenta il loro commitment che le porterà sempre più verso la strada, prima di progenitori, poi di precursori ed infine verso lo stato di cellula unipotente. Lungo questo cammino è perciò possibile osservare una popolazione cellulare con vari gradi di staminalità, vale a dire con vari gradi di differenziazione (Fig. 1).

Un fattore di primo piano nel mantenimento della popolazione staminale e nella regolazione della differenziazione in vivo è il microambiente in cui è sita la cellula staminale stessa e che prende il nome di nicchia staminale (o stem cell niche). Nella nicchia di appartenenza le cellule staminali mantengono il self-renewal e sono mantenute quiescenti in uno stato indifferenziato (con self-renewal si intende la capacità di originare una identica fenocopia della cellula staminale, ossia almeno una delle due cellule figlie deve avere capacità di self-renewal e potenza differenziativa tali e quali a quelle della cellula madre).

La nicchia staminale riveste quindi un ruolo chiave per l'equilibrio tra quelli che sono due poli opposti come autorinnovamento e differenziazione. Uno sbilanciamento potrebbe indirizzare verso un eccessivo self-renewal portando a un'espansione cellulare abnorme, evento che incanala verso la tumorigenesi; mentre una eccessiva differenziazione condurrebbe verso una deplezione del compartimento di ASC, con il rischio di incapacità di mantenimento dell'omeostasi tissutale e di ricambio cellulare. 


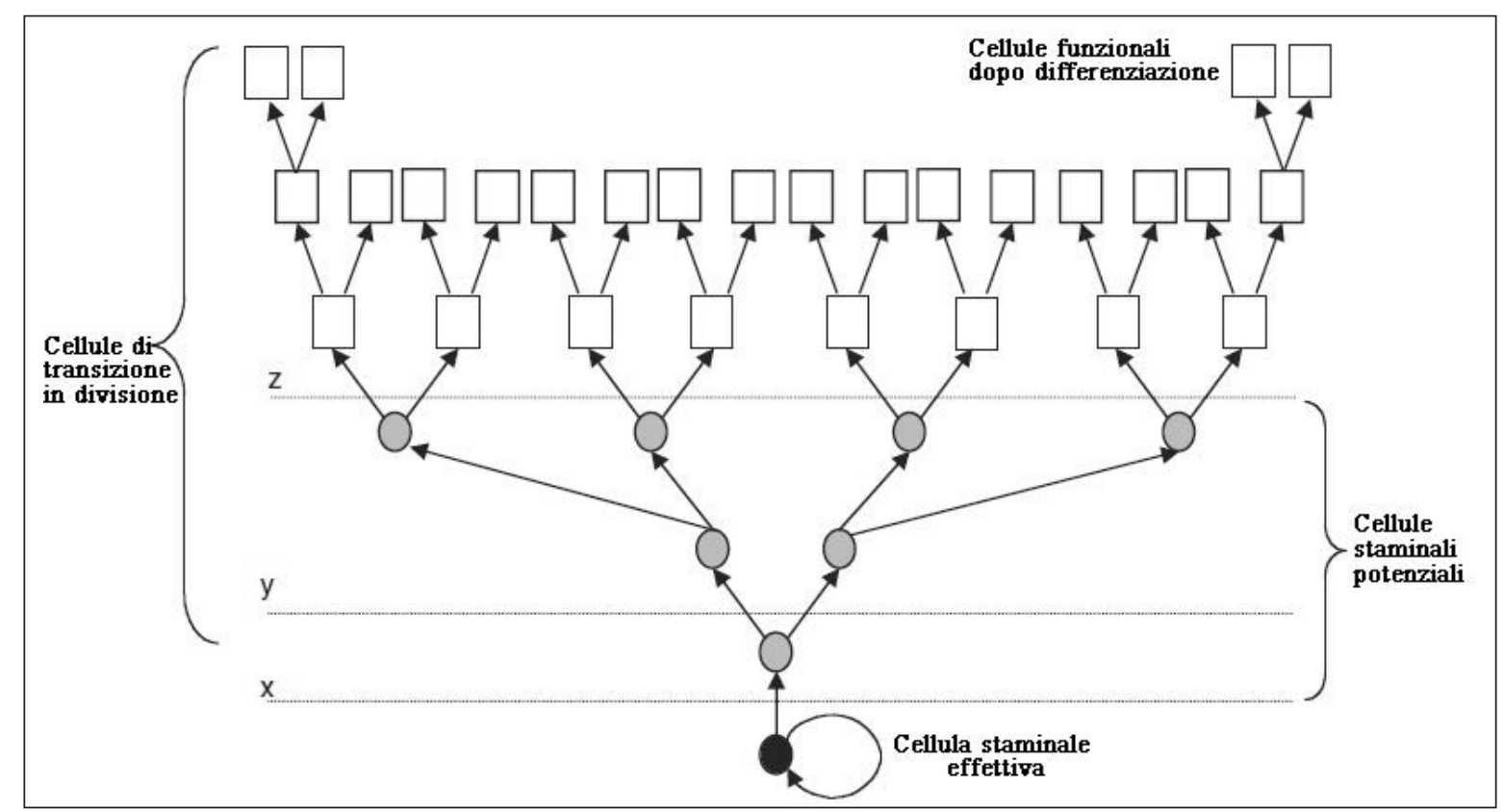

Fig. 1 - Schematizzazione del processo differenziativo di una cellula staminale adulta. Dirigendosi verso cellula specializzata la cellula staminale prolifera dando luogo a una popolazione di transizione con caratteristiche intermedie tra l'ASC e la cellula terminalmente differenziata. Nella popolazione di transizione rientrano i progenitori staminali, i quali discendono da cellule staminali ma sono più ristretti nel loro potenziale differenziativo e nella capacità di self-renewal (1).

All'interno della nicchia staminale due meccanismi permettono la creazione di cellule figlie che seguiranno destini diversi: difatti mentre una cellula essenzialmente rimarrà allo stato indifferenziato, l'altra andrà incontro a specializzazione. In questo modo è mantenuta la popolazione staminale ma allo stesso tempo sono fornite unità commissionate pronte alla sostituzione. Questi due meccanismi sono rappresentati dalla divisione asimmetrica pre-divisionale, la quale permette alle cellule di avere due diversi sorti nonostante le cellule restino nello stesso microambiente, e dalla divisione asimmetrica post-divisionale, la quale mette le due cellule figlie in due microambienti diversi grazie a un differente orientamento del piano di divisione relativamente alla nicchia (all'interno della nicchia sono ad esempio mantenuti il self renewal e le caratteristiche di staminalità) (Fig. 2).

La divisione asimmetrica è denominata pre-divisionale qualora mRNA e proteine commissionanti siano ripartiti in modo non eguale tra le due cellule figlie, facendo sì che con la mitosi avvenga essenzialmente il differenziamento. È invece detta asimmetrica post-divisionale quando sono create due cellule figlie identiche che sono però esposte a segnali estrinseci diversi, forniti da distinti microambienti locali. Alla fine di questo processo avremo perciò una cellula che rimanendo nella nicchia

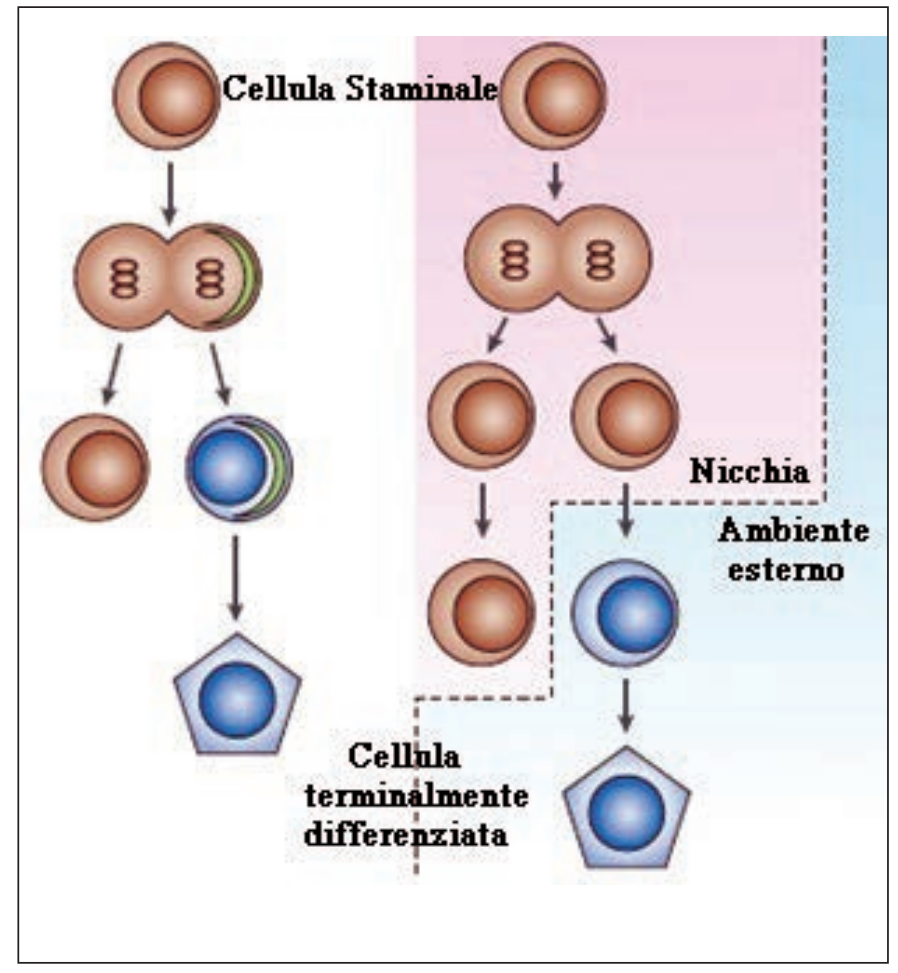

Fig. 2 - Schematizzazione concettuale della divisione asimmetrica pre-divisionale (sinistra) e post-divisionale (destra) (1). 
mantiene le sue caratteristiche di staminalità, e un'altra che va incontro a specializzazione. Il fuso mitotico in questo caso sarà posizionato di modo che una delle due cellule perda il contatto con le cellule di supporto che costituiscono la nicchia staminale stessa.

Mentre la divisione asimmetrica pre-divisionale è spesso osservabile nelle cellule staminali del primo sviluppo embrionale, le quali adottano tale tipologia di divione con la finalità di incrementare le dimensioni del corpo; la divisione asimmetrica post-divisonale è invece maggiormente riscontrabile nelle cellule staminali permanenti del tardo stadio evolutivo dell'embrione e nelle cellule staminali adulte che presumibilmente originano da queste. In tal caso lo scopo è ovviamente quello di mantenere l'integrità tissutale.

\section{Conclusioni}

Lo studio della potenza di una cellula è sicuramente di grande interesse soprattutto in ambito terapeutico, sia perché ciò permette di andare ad individuare l'origine di quelle entità staminali che mantengono la funzionalità di un tessuto o lo riparano in seguito a un insulto, sia poiché individuando e soprattutto modulando le capacità differenziative di tali cellule sarà possibile, in un prossimo futuro, ottenere un indirizzamento verso il lineage desiderato. Questo assume rilevanza soprattutto in ottica dell'impiego di cellule staminali embrionali, le quali se inoculate in vivo molto spesso sono capaci di dare origine allo sviluppo di teratomi, motivo per cui molta della ricerca in campo della rigenerazione tissutale si è ad oggi spostata verso le cellule staminali adulte. Grazie però ad uno studio sempre più approfondito sulle caratteristiche e sui marcatori che individuano cellule con più di una possibile specializzazione, stanno venendo acquisite sempre maggiori informazioni e, ovviamente, maggiori possibilità di utilizzo per tutti i tipi di cellula staminale.

\section{Indirizzo degli Autori: \\ Duccio Lombardi \\ Via B. Scala 23 \\ 50126 Firenze \\ lombarduccio@alice.it}

\section{Bibliografia}

1. Lanza R. Gearhart J. Hogan B. Melton D. Pedersen R. Thomson J. West M. Handbook of Stem Cells 2004, Elsevier Inc. Academic Press.

2. Takahashi K. Yamanaka S. Induction of pluripotent stem cells from mouse embryonic and adult fibroblast cultures by defined factors. Cell 2006; 126(4): 663-76.

3. Ohnuki M. Takahashi K. Yamanaka S. Generation and characterization of human induced pluripotent stem cells. Curr Protoc Stem Cell Biol 2009; Chapter 4: Unit 4A.2. 